\title{
Sexually dimorphic genetic architecture of complex traits in a large-scale $F_{2}$ cross in pigs
}

\author{
Leilei Cui, Junjie Zhang, Junwu Ma, Yuanmei Guo, Lin Li, Shijun Xiao, Jun Ren, Bin Yang* and Lusheng Huang
}

\begin{abstract}
Background: It is common for humans and model organisms to exhibit sexual dimorphism in a variety of complex traits. However, this phenomenon has rarely been explored in pigs.

Results: To investigate the genetic contribution to sexual dimorphism in complex traits in pigs, we conducted a sex-stratified analysis on 213 traits measured in 921 individuals produced by a White Duroc $\times$ Erhualian $\mathrm{F}_{2}$ cross. Of the 213 traits examined, 102 differed significantly between the two sexes (q value $<0.05$ ), which indicates that sex is an important factor that influences a broad range of traits in pigs. We compared the estimated heritability of these 213 traits between males and females. In particular, we found that traits related to meat quality and fatty acid composition were significantly different between the two sexes, which shows that genetic factors contribute to variation in sexual dimorphic traits. Next, we performed a genome-wide association study (GWAS) in males and females separately; this approach allowed us to identify $13.6 \%$ more significant trait-SNP (single nucleotide polymorphism) associations compared to the number of associations identified in a GWAS that included both males and females. By comparing the allelic effects of SNPs in the two sexes, we identified 43 significant sexually dimorphic SNPs that were associated with 22 traits; 41 of these 43 loci were autosomal. The most significant sexually dimorphic loci were found to be associated with muscle hue angle and Minolta $a^{*}$ values (which are parameters that reflect the redness of meat) and were located between 9.3 and $10.7 \mathrm{Mb}$ on chromosome 6. A nearby gene i.e. NUDT7 that plays an important role in heme synthesis is a strong candidate gene.
\end{abstract}

Conclusions: This study illustrates that sex is an important factor that influences phenotypic values and modifies the effects of the genetic variants that underlie complex traits in pigs; it also emphasizes the importance of stratifying by sex when performing GWAS.

\section{Background}

Males and females differ in many aspects, from their chromosomes (X vs. Y) to their body morphologies to their reproductive behaviors. In model organisms such as mice and Drosophila, many traits ranging from gene expression levels [1] to embryonic development [2] have been reported to show sexual differences. Furthermore, the prevalence and severity of many common diseases in humans, such as type 2 diabetes [3] and asthma [4], also vary between men and women.

The fact that a broad range of complex traits are sexually dimorphic indicates that males and females are characterized by important biological differences, which

\footnotetext{
* Correspondence: binyang@live.cn

Key Laboratory for Animal Biotechnology of Jiangxi Province and the Ministry of Agriculture of China, Jiangxi Agricultural University, 330045 Nanchang, China
}

can modify the effects of genes on complex traits. Understanding sex-dependent differences in the genetic architecture of complex traits can help to generate insights into the evolution of males and females; it can also help in the development of sex-specific medical treatments in humans and breeding objectives in farm animals. Many traits in humans, such as gene expression levels [5], blood metabolite levels [6], and the prevalence of common diseases [7], have been reported to have sexually dimorphic genetic architectures. Similar situations have also been documented in model organisms such as mice (e.g., fat mass [8]) and Drosophila (e.g., gene expression [9]), which indicates that sexually dimorphic genetic control of complex traits is common across species. In contrast, the molecular basis of sex-specific genetic regulation of complex traits in pigs remains less explored. 
Genome-wide association studies (GWAS) have been used to identify genetic loci that affect complex traits in pigs, such as traits related to body composition [10], hematology [11], and disease susceptibility [12]. In a previous study, we conducted a large-scale White Duroc $\times$ Chinese Erhualian $\mathrm{F}_{2}$ cross, measured a variety of traits and obtained $60 \mathrm{~K}$ genotypes from more than $1000 \mathrm{~F}_{2}$ individuals. Using these data, we performed GWAS to identify the loci associated with various traits $[13,14]$. However, our previous study and most other GWAS in pigs did not explicitly consider the possibility of sex-specific differences in the genetic architecture underlying the traits examined. The associations between single nucleotide polymorphisms (SNPs) and traits were simply assessed using linear models that treated sex as a fixed effect, which could have reduced the power to detect loci that demonstrated sex-specific differences.

In this study, we used a sex-stratified GWAS to examine sex-specific differences in gene regulation using 213 traits in individuals from our large $F_{2}$ pig experimental population. Data were obtained from a total of $921 \mathrm{~F}_{2}$ individuals of both sexes. All the male pigs were castrated when they were 90 days old; therefore, the influence of sex hormones was limited in this study. First, we evaluated sex-specific differences in the 213 traits using a linear regression model. Next, by comparing the genome-wide SNP-trait association signatures found in males versus females, we identified loci that displayed sexually dimorphic expression; these loci were associated with a variety of traits related to meat quality, fatty acid composition, food intake, and hematological parameters. This study emphasizes that sex is an important factor that can modify the effects of the genetic variants underlying complex traits in pigs.

\section{Methods}

\section{Animals}

All the pigs used in this study were the product of a largescale White Duroc $\times$ Erhualian $\mathrm{F}_{2}$ cross; the design and management of this experimental population have been described elsewhere [15]. Briefly, two White Duroc boars were mated to 17 Erhualian sows to generate the $F_{1}$ pigs. Then, nine $F_{1}$ boars were crossed with $59 \mathrm{~F}_{1}$ sows to produce $1912 \mathrm{~F}_{2}$ pigs (in six batches). All the $F_{2}$ animals were raised at the Jiangxi Agricultural University experimental farm (Nanchang, China); they were fed ad libitum and weaned at the age of 46 days. The male pigs were castrated at the age of 90 days. The castrated males and the females lived in mixed pens and were treated equally throughout the experiment. All the $\mathrm{F}_{2}$ pigs were slaughtered at the age of $240 \pm 3$ days; overall, we measured more than 400 traits in the $F_{2}$ population. We obtained $60 \mathrm{~K} \mathrm{SNP}$ genotypes from $1000 \mathrm{~F}_{2}$ pigs using porcine SNP60 Beadchips (Illumina). The procedures used to phenotype the pigs and obtain the $60 \mathrm{~K}$ genotypes have been described in previous publications [13-17]. All animal work was conducted according to the guidelines for the care and use of experimental animals established by the Ministry of Agriculture of China.

\section{Data used}

We investigated 213 traits in the $F_{2}$ pigs produced by a large-scale White Duroc $\times$ Chinese Erhualian $\mathrm{F}_{2}$ cross. These traits are related to a broad range of characteristics, including growth, fatness, meat quality, hematological parameters, and serum glucose and lipid levels (See Additional file 1: Table S1). For the 213 traits examined, we obtained both phenotypic values and $60 \mathrm{~K}$ SNP genotypes for between 217 and 919 pigs; we had both sets of data for at least 100 individuals for both sexes.

\section{Quality control of 60 K SNP genotype data}

The quality control procedure used for the $60 \mathrm{~K} \mathrm{SNP}$ genotype data and the details of the GWAS are the same as previously described [14]. Briefly, we retained samples with SNP call rates greater than $10 \%$ and Mendelian inconsistence rates smaller than 0.05; we kept SNPs with call rates higher than 0.9 , minor allele frequencies higher than $0.05, P$ values greater than $10^{-6}$ for the Hardy-Weinberg equilibrium test and Mendelian error rates smaller than 0.1 . These procedures were implemented in Plink v1.07 [18]. In total, 39454 SNPs and 921 individuals were used in the subsequent analyses.

\section{Statistical analyses}

We log-transformed $\left(\log _{2}\right)$ the phenotypic values of any traits that strongly deviated from normality (Shapiro test $P$ value $\left.<10^{-8}\right)$. We assessed the significance of the effects of sex on the 213 traits examined using the $l m$ function in R [19]; batch was included as a fixed effect. To evaluate sex-specific differences in the genetic architecture of the 213 traits, we conducted a sex-stratified analysis in which we estimated trait heritability and performed separate GWAS for males versus females. We used the R package GenABEL [20]; batch was included as a fixed effect. Heritability was estimated based on a kinship matrix calculated from the $60 \mathrm{~K}$ SNP genotype data and using the polygenic function. The associations between the SNP genotypes and the traits were evaluated using mixed linear models (mmscore function). As in the previously described GWAS, sex and batch were fitted as fixed effects. We assessed the empirical significance of the difference in heritability between the two sexes using a permutation test [21]: for each trait, we shuffled the gender labels of individuals 1000 times to generate a null distribution of absolute differences in heritability between the two sexes. We evaluated the $P$ values of the associations yielded by the GWAS after applying a Bonferroni correction. The suggestive significance level was set to $1 / \mathrm{N}_{\text {snp }}$, where $\mathrm{N}_{\text {snp }}$ was the number of SNPs tested in the GWAS; this threshold allowed one false 
positive association per genome scan. The genome-wide significance level was set to $0.05 / \mathrm{N}_{\mathrm{snp}}$.

To identify genetic variants associated with strong sexual dimorphism, we tested for sex-specific differences in SNP effects using an approach similar to the one described in [6]. Briefly, we calculated the z-statistics as follows:

$$
Z=\frac{\beta_{\text {male }}-\beta_{\text {female }}}{\sqrt{s e\left(\beta_{\text {male }}\right)^{2}+s e\left(\beta_{\text {female }}\right)^{2}}},
$$

where $\beta_{\text {male }}$ and $\beta_{\text {female }}$ are the SNP effects estimated in males and females, respectively, and $s e\left(\beta_{\text {male }}\right)$ and $s e$ $\left(\beta_{\text {female }}\right)$ are the standard errors of those SNP effects. Given the null hypothesis that SNP effects in males and female were equal, the $\mathrm{z}$-statistic has a standard normal distribution. Single-tailed tests were used to evaluate the significance of any sex-specific difference. The significance level was set using the Bonferroni approach described above. To avoid spurious signals caused by low minor allele frequencies (MAF) in either sex, we exclude SNPs with MAF values of less than $5 \%$ (in males or females).

\section{Results}

\section{Effect of sex on the traits studied}

We used a simple linear regression model to look for sexspecific differences in phenotypic values. Of the 213 traits examined, 102 showed significant sex-specific differences (q value $[22]<0.05$ ). Out of these 102 traits, 20 had q values greater than $1 \times 10^{-4}$; these traits were related to fatness, fatty acid composition, growth and meat $\mathrm{pH}$ values (See Additional file 2: Table S2). Generally, males had greater abdominal fat weight (Figure 1A), greater intramuscular fat content (Figure 1B), greater leaf fat weight and higher levels of saturated fatty acids in both their fat and muscle tissues. Abdominal fat weight appeared to be the most significant sexually dimorphic trait $\left(\mathrm{q}\right.$ value $\left.=7.9 \times 10^{-13}\right)$; on average, males had $72 \mathrm{~g}$ more abdominal fat than did females (Figure 1A). Moreover, the meat of male pigs had a higher $\mathrm{pH}$. In contrast, females had higher levels of unsaturated fatty acids and a higher average daily gain from 21 to 46 days old $\left(\mathrm{q}\right.$ value $=1 \times 10^{-5}$ ) (Figure $1 \mathrm{C}$ and $\left.\mathrm{D}\right)$. Males and females showed a range of differences (from 0.74 to 1.58 fold) in their phenotypic values; most traits differed by more than $50 \%$ between the two sexes (See Additional file 2: Table S2).

\section{Heritability in males versus females}

We also estimated the heritability of the 213 traits examined using separate male and female kinship matrices constructed using the autosomal $60 \mathrm{~K}$ SNP genotype data (see Methods section). Trait heritability was significantly correlated between males and females (Spearman correlation $\mathrm{r}=0.65, P=3.94 \times 10^{-27}$ ) (See Additional file 3: Table S3) (Figure 1E). We found 24 traits that showed significant sex-specific differences in heritability (empirical $P$ value $<0.05)$. For instance, meat quality, forward gait score at 223 days old, platelet distribution width and serum glucose levels were more heritable in males than in females (See Additional file 3: Table S3) (Figure 1E). In contrast, fatty acid composition, weight at 46 days old and skin thickness were more heritable in females (See Additional file 3: Table S3) (Figure 1E). A trait with greater heritability is a trait for which a greater degree of phenotypic variance can be accounted for by genetic variants. This result indicates the importance of the sexually dimorphic genetic architecture that underlies these traits in pigs.

\section{Identifying sexually dimorphic loci associated with phenotypic traits}

To investigate the genetic basis of sexual dimorphism of traits, we performed a sex-stratified GWAS and assessed sex-specific differences in the allelic effects at each SNP locus using z-statistics [6] (see Methods section); this analysis revealed the extent of sex-specific genetic effects of particular SNPs on target traits. A total of 22 traits related to meat quality, fatty acid composition, hematological parameters and food intake were controlled by 43 significant $\left(P<2.5 \times 10^{-5}\right)$ sexually dimorphic loci (See Additional file 4: Table S4). Of these 43 loci, 41 (95.3\%) were on autosomes. The most significant signatures $\left(P=4.8 \times 10^{-7}\right)$ were detected for hue angle $\left(\mathrm{H}^{*}\right)$ value at 45 minutes post-slaughter (Figure 2A). Hue angle is a meat color parameter related to Minolta $\mathrm{b}^{*}$ (yellowness) and $\mathrm{a}^{*}$ (redness) values, which can be calculated as $\mathrm{H}^{*}=\arctan$ $\left(\mathrm{b} * / \mathrm{a}^{*}\right)$. This locus exhibited a significant male-specific association (Figure 2B) but negligible association in females (Figure 2D). It should be noted that this locus was not detected in the GWAS that included both males and females (combined GWAS). This locus also showed a male-specific association with Minolta $\mathrm{a}^{*}$ at 45 minutes post-slaughter, which is another meat redness parameter. Other notable traits associated with significant sexually dimorphic loci include stearic acid (C18:0) levels in muscle, food intake and mean corpuscular hemoglobin concentrations (see Additional file 4: Table S4).

We compared the significant signals obtained in the sex-stratified GWAS with the significant signals obtained in the combined GWAS. Between the sex-stratified GWAS and the combined GWAS, we identified a total of 16996 significant SNPs $\left(P\right.$ value $\left.<2.5 \times 10^{-5}\right)$ associated with the 213 traits. Of these SNPs, $70.5 \%$ were detected by both approaches. Notably, 2035 significant SNPs were detected only by the sex-stratified GWAS; this corresponds to $13.6 \%$ of the loci detected by the combined GWAS (Figure 3A). As a consequence, this result suggests 


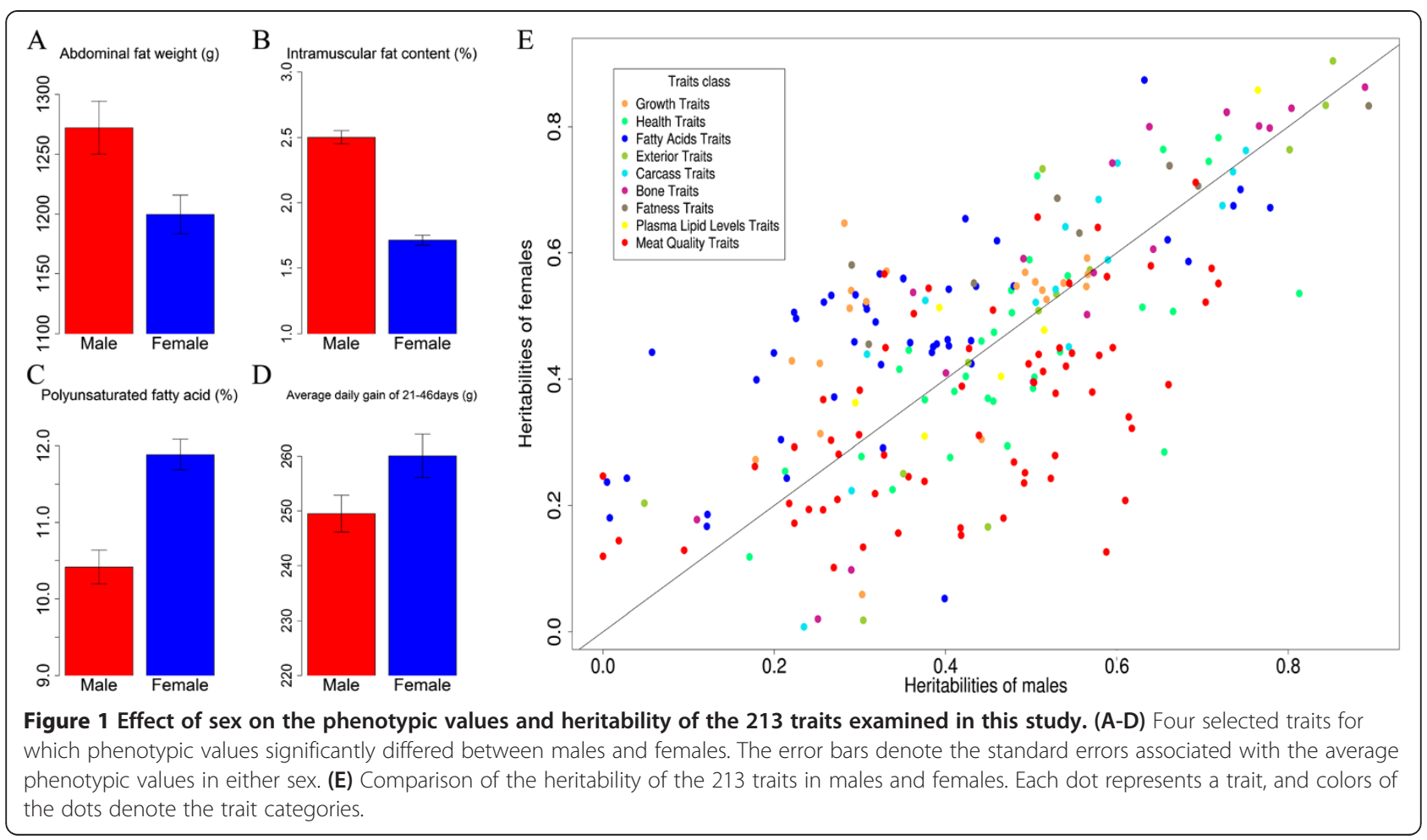

that $13.6 \%$ more loci can be identified by using a sexstratified GWAS in addition to a combined GWAS. We found that the values of the z-statistics associated with the new SNPs revealed by the sex-stratified GWAS were located in the tail of z-statistic distribution (Figure 3B) $(t$ test $P$ value $=2.3 \times 10^{-221}$ ). This finding indicates that most of these new SNPs have sex-specific effects. Therefore, their signatures were weakened in the combined GWAS.

\section{Discussion}

GWAS have become a powerful tool for detecting genetic variants associated with complex traits in pigs. Although studies in humans and model organisms have demonstrated the importance of sexual dimorphism in the genetic architecture of complex traits, to date nearly all the GWAS performed in pigs have ignored sex-specific effects or sex-by-genotype interactions, which means that they have possibly missed an opportunity to identify genetic variants that act differently in males and females.

To study sex-specific genetic effects on complex traits in pigs, we conducted a sex-stratified GWAS using 921 (507 males +414 females) $\mathrm{F}_{2}$ pigs produced by a White Duroc $\times$ Erhualian $F_{2}$ cross. In contrast to human GWAS, the pigs in this GWAS were raised under the same controlled conditions and were fed a uniform diet; furthermore, most traits were phenotypically characterized at the same time (after slaughter). These experimental features have largely reduced the level of environmental noise and increased the ability to detect sexually dimorphic loci.
Indeed, we were able to identify many extremely significant loci associated with traits related to meat quality [16], fatty acid composition [14], hematological parameters [17] and blood lipid levels [13], which suggests that the data obtained in this study can contribute to the identification of sex-specific genetic effects on complex traits.

Sex hormones are important factors that mediate sexually dimorphic gene expression in mice [23]. It should be noted that all the male pigs in this study were castrated when they were 90 days old. Therefore, the sex-specific effects identified in this study are less likely to have been caused by gonadal hormones than by sex chromosome dosage compensation or other unknown factors.

Of the 213 traits examined, 102 demonstrated significant sex-specific differences. Some of these traits were related to growth, fatness, meat quality, serum lipid levels and hematological parameters. These results indicate that sex is an important factor that affects the phenotypic values of various traits in pigs, even in the absence of the influence of sex hormones. Some traits showed a highly significant degree of sexual dimorphism (q value $<10^{-4}$ ); for instance, we found that the castrated males studied in our study tended to accumulate more abdominal fat, leaf fat and intramuscular fat and that they had higher levels of saturated fatty acids in their fat and muscle tissue than females. From the standpoint of food quality, this result indicates that pork from castrated male pigs might taste better since IMF content is related to meat juiciness and flavor. In contrast, females grew faster during the 

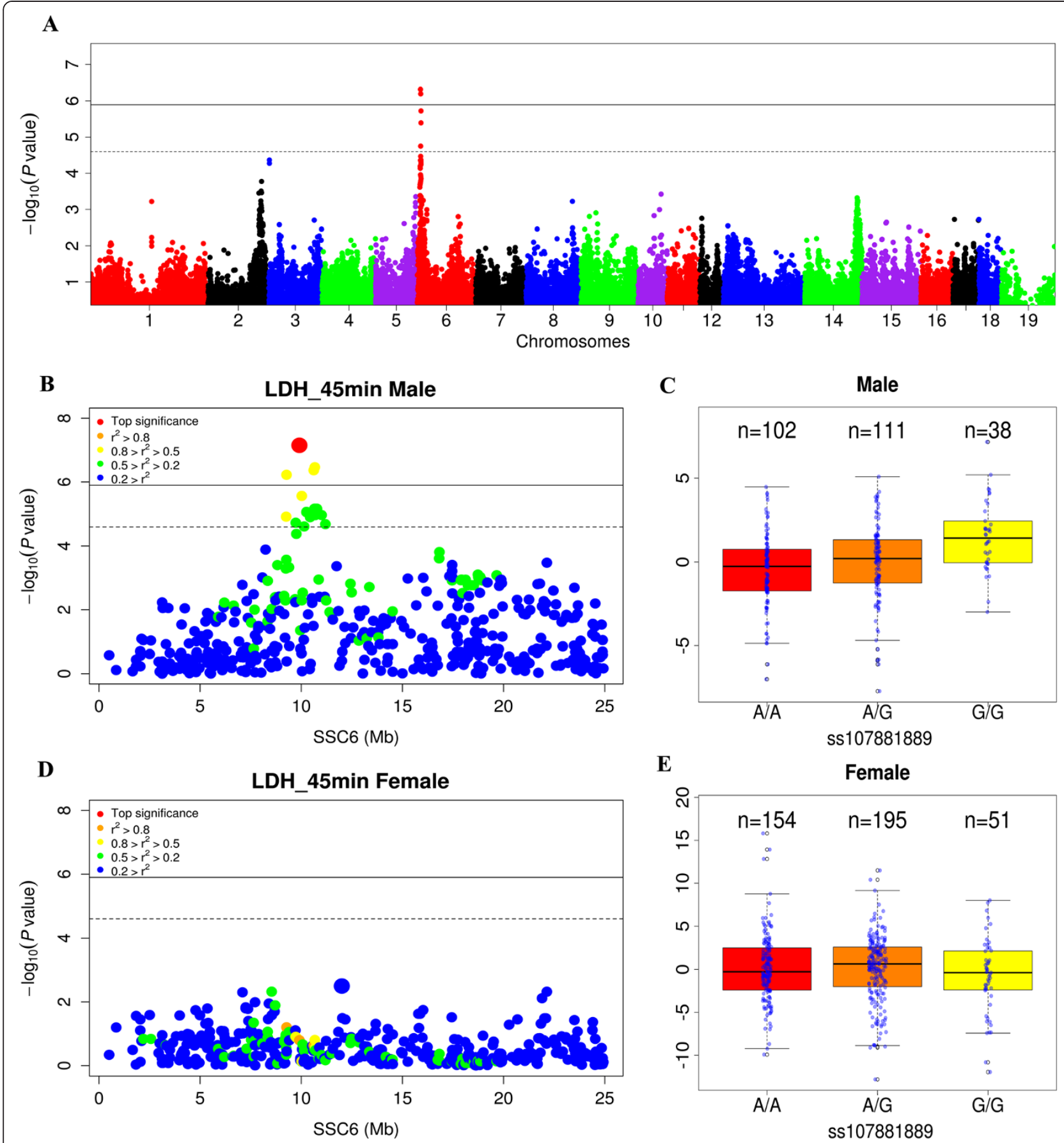

Figure 2 Identification of sexually dimorphic loci using a sex-stratified GWAS. (A) Manhattan plot of sex-specific signals; the significance of the sex-specific effects ( $-\log _{10} P$ value) were plotted against the loci's genomic positions. The dashed and solid horizontal lines represent suggestive and genome-wide significance thresholds. (B) and (D) Regional plots of GWAS signals on chromosome 6 for males (B) and females (D). (C) and (D) Distribution of phenotypic values for the three SNP genotypes most associated with the longissimus muscle hue angle values in males (C) and females (E).

early stages of life (from 21 to 46 days) and had higher levels of unsaturated fatty acids in their tissues; this result indicates that pork from female pigs might be healthier since foods containing a higher proportion of unsaturated fatty acids are better for human health
[24]. These results support that sex does influence a broad range of traits in pigs.

Using genome-wide SNP genotypes to estimate the heritability of complex traits has proven to be feasible [25]. Furthermore, genetic component analyses using 


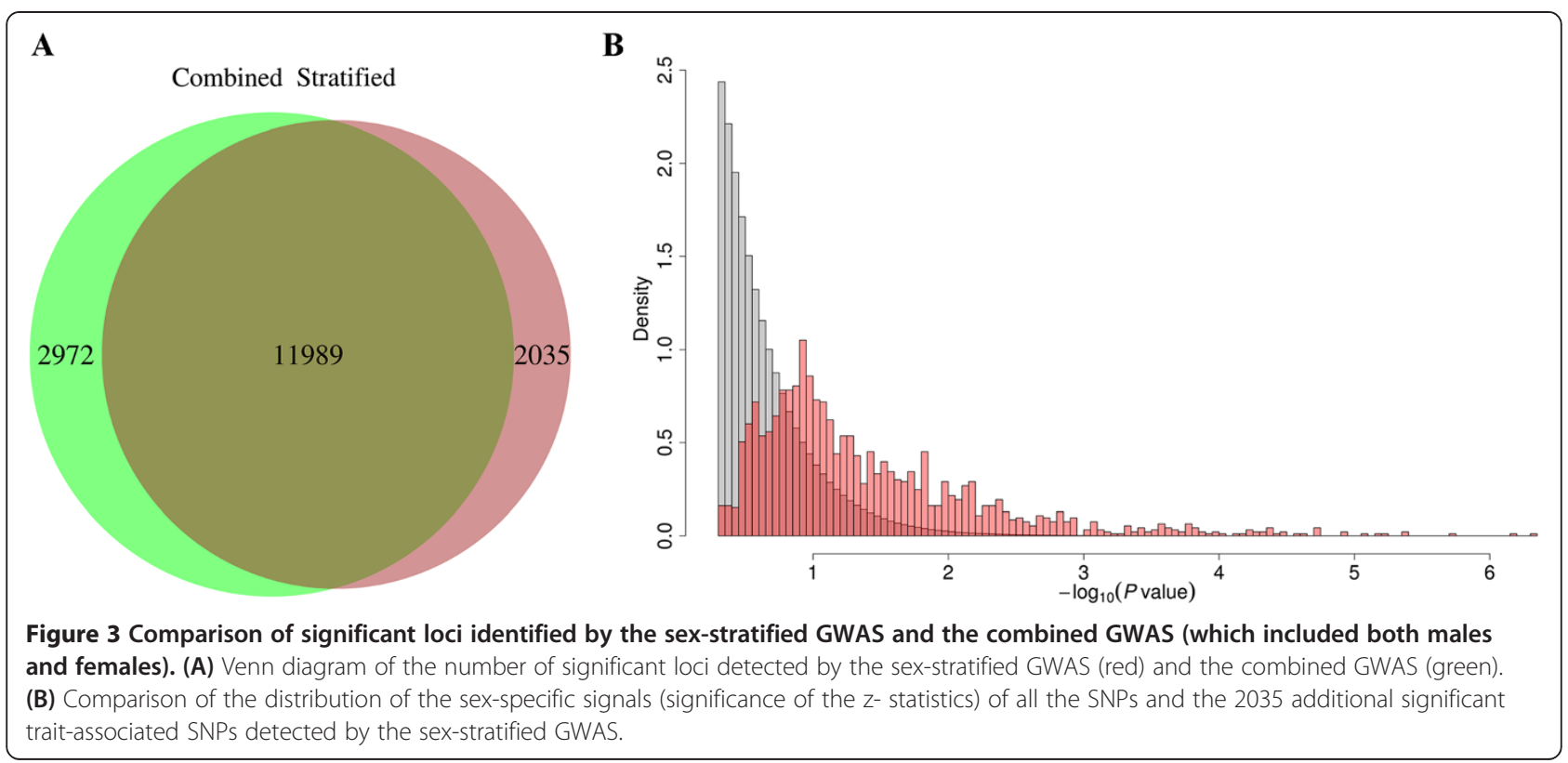

pedigree data have illustrated that significantly different types of genetic architectures underlie multiple traits related to body composition in humans [26]. However, few studies have examined differences in the heritability of complex traits between males and females using genotype data. Here, we estimated trait heritability separately in males and females using $60 \mathrm{~K} \mathrm{SNP}$ genotypes. We found that the heritability of 22 traits differed significantly between males and females; some of these traits are related to waist vertebrae number, growth at an early age, platelet distribution width and serum glucose levels. Remarkably, we also found clusters of traits related to fatty acid composition and meat quality. Setting statistical significance aside, we found that SNP-genotype-based heritability was higher in females than in males for 35 (79.5\%) of the 44 traits related to the fatty acid composition of fat or muscle tissues and for all of the fatness traits (Figure 1E) (see Additional file 3: Table S3). This finding indicates that genetic variants accounted for more of the variance in traits related to fatty acid composition and fatness in females than in males. In contrast, the heritability of most of the meat quality traits including temperature, $\mathrm{pH}$, and the color of longissimus muscle and semimembranosus muscle at various time points postslaughter was higher in males than in females (Figure 1E) (see Additional file 3: Table S3). Although these results could be biased by the presence of correlations between traits, they nonetheless illustrate that the genetic architecture that underlies certain categories of traits differed between the two sexes. This is possibly due to different evolutionary pressures imposed by natural and artificial selection that act to alter the molecular pathways that shape these traits in males versus females.
Compared to the combined GWAS (which included both males and females), the sex-stratified GWAS identified $13.6 \%$ more loci associated with the traits. This percentage of novel loci identified by the sex-stratified GWAS is similar to what has been found in studies of gene expression traits in humans [5]. This result illustrates the importance of performing sex-stratified GWAS or including a sex-bygenotype interaction in routine GWAS.

To identify sexually dimorphic loci associated with the traits being examined, we scanned the whole genome to compare the effects of SNPs in the two sexes. The most significant sexually dimorphic loci were found between 9.3 and $10.7 \mathrm{Mb}$ on chromosome 6; they were associated with hue angle and Minolta $\mathrm{a}^{*}$ values at $45 \mathrm{~min}$ post slaughter, which are color parameters that reflect meat redness. Meat color is an important feature for consumers since it reflects meat quality and freshness. It has been reported that $\sim 90 \%$ of the variance in the color parameters of longissimus muscle in pigs can be explained by pigment content and myoglobin forms [27]. Moreover, the Minolta $a^{*}$ value has been reported to be significantly correlated with myoglobin expression in muscle in Pietrain pigs [28]. Presumably, the underlying genetic variants could affect pigment content or myoglobin content, which in turn could affect meat color. Using UCSC online gene annotation tools, we searched for candidate genes in a $1-\mathrm{Mb}$ region around the SNPs that were significantly associated with hue angle values; the nudix (nucleoside diphosphate linked moiety X)-type motif 7 gene (NUDT7), located at $10.1 \mathrm{Mb}$ on chromosome 6 is of particular interest. This gene plays an important role in heme biosynthesis, and overexpression of NUDT7 has been associated with reduced heme biosynthesis in pig skeletal muscle [29]. In 
mice, the hypomethylation of NUDT7 in female offspring can be induced by high levels of maternal folic acid during gestation [30], which indicates that sex-specific epigenetic modification of this gene might be responsible for the sex-specific signals detected by the GWAS.

We found that 41 (95.3\%) of the 43 significant sexually dimorphic loci were autosomal. This result is not unexpected since many health-related traits, such as fat distribution in humans, have also been observed to be associated with sexually dimorphic loci located on autosomes [31]. The molecular mechanisms that underlie these autosomal sexually dimorphic loci are not clear. Possible explanations are that sex-specific effects could contribute to heritable epigenetic differences between males and females [32] or that these differences are mediated by the different sex chromosomes found in males versus females.

We checked whether the 22 traits that were associated with significant sexually dimorphic loci demonstrated significantly different phenotypic values for males versus females. We found that $12(54.4 \%)$ of the 22 traits did not have sex-specific phenotypic values $(\mathrm{q}>0.05)$. However, not one of the top five traits i.e. abdominal fat weight; C16:1, C18:0 and C20:3 levels in abdominal fat; and the saturated fatty acid content of muscle that demonstrated sex-specific phenotypic values $\left(\mathrm{q}<10^{-4}\right)$ were associated with significant sexually dimorphic loci. These results suggest that even if phenotypic values differ significantly between the two sexes, it does not necessarily mean that strong sex-specific genetic controls are in place, and vice versa. Similar results have been reported for gene expression traits in humans [5].

\section{Conclusions}

In this study, we looked for evidence of sexual dimorphism in the phenotypic values of and the genetic variants associated with 213 traits related to a broad range of pig traits. Some of these traits, namely those related to growth, fatness, meat quality and palatability, are of economic importance. The results of this study have improved our understanding of the relationship between sex and complex traits. About half of the 213 traits examined in this study showed significant sex-specific differences in their phenotypic values, which demonstrates that sex is an important factor that influences the phenotypic values of quantitative traits in pigs even when the influence exerted by sex hormones is limited. The sex-stratified GWAS revealed the existence of a landscape of sexspecific genetic effects on a variety of pig traits and demonstrated that sex-specific regulation is an important aspect of the genetic architecture that underlies complex traits. Our results suggest that future GWAS should stratify their analyses by sex or incorporate a sex-by-genotype interaction to identify sexually dimorphic loci associated with complex traits in pigs and other organisms.

\section{Additional files}

\begin{abstract}
Additional file 1: Table S1. Title: Descriptive statistics for the 213 trait examined in this study. Description: This file contains the abbreviation and description of 213 traits, and their descriptive statistics including mean, standard deviation, sample size and estimated heritability.
\end{abstract}

Additional file 2: Table S2. Title: The 102 traits in this study that displayed significant sex-specific phenotypic values $(q<0.05)$. Description: This file provides detailed statistical results on 102 traits that displayed sex dimorphic phenotype values.

Additional file 3: Table S3. The sex-specific differences in the estimated heritabilities of the 213 traits examined in this study. Description: This file lists the estimated heritabilities of 213 traits for males and females, absolute difference between heritabilities of males and females, 5\% significance thresholds of absolute difference between heritabilities of males and females obtained by 1000 permutation test.

Additional file 4: Table S4. Significant sexually dimorphic loci associated with 24 traits identified in this study. Description: This file contains detailed information on 43 significant sexually dimorphic loci for 24 traits.

\section{Competing interests}

The authors declare that they have no competing interests.

\section{Authors' contributions}

LH conceived and designed the experiments and revised the manuscript; BY analyzed the data and wrote the manuscript; LC analyzed the data and wrote part of the manuscript; JZ analyzed part of the data; JM, YG, SX, and LL organized and participated in the phenotype measurements. JR helped with data analysis. All authors read and approved the final manuscript.

\section{Acknowledgments}

The work was funded by the Natural Science Foundation of China (31201776).

Received: 17 May 2014 Accepted: 20 October 2014

Published online: 06 November 2014

\section{References}

1. Ayroles JF, Carbone MA, Stone EA, Jordan KW, Lyman RF, Magwire MM, Rollmann SM, Duncan LH, Lawrence F, Anholt RR, Mackay TF: Systems genetics of complex traits in Drosophila melanogaster. Nat Genet 2009, 41:299-307.

2. Seller MJ, Perkins-Cole KJ: Sex difference in mouse embryonic development at neurulation. J Reprod Fertil 1987, 79:159-161.

3. Woods SC, Gotoh K, Clegg DJ: Gender differences in the control of energy homeostasis. Exp Biol Med 2003, 228:1175-1180.

4. Chen $Y$, Stewart $P$, Johansen H, McRae L, Taylor G: Sex difference in hospitalization due to asthma in relation to age. J Clin Epidemio/ 2003, 56:180-187.

5. Dimas AS, Nica AC, Montgomery SB, Stranger BE, Raj T, Buil A, Giger T, Lappalainen T, Gutierrez-Arcelus M, MuTHER Consortium, McCarthy MI, Dermitzakis ET: Sex-biased genetic effects on gene regulation in humans. Genome Res 2012, 22:2368-2375.

6. Mittelstrass K, Ried JS, Yu Z, Krumsiek J, Gieger C, Prehn C, Roemisch-Margl W, Polonikov A, Peters A, Theis FJ, Meitinger T, Kronenberg F, Weidinger S, Wichmann HE, Suhre K, Wang-Sattler R, Adamski J, Illig: Discovery of sexual dimorphisms in metabolic and genetic biomarkers. PLOS Genet 2011, 7:e1002215.

7. Ober C, Loisel DA, Gilad Y: Sex-specific genetic architecture of human disease. Nat Rev Genet 2008, 9:911-922.

8. Wang S, Yehya N, Schadt EE, Wang H, Drake TA, Lusis AJ: Genetic and genomic analysis of a fat mass trait with complex inheritance reveals marked sex specificity. PLoS Genet 2006, 2:e15.

9. Massouras A, Waszak SM, Albarca-Aguilera M, Hens K, Holcombe W, Ayroles JF, Dermitzakis ET, Stone EA, Jensen JD, Mackay TF, Deplancke B: Genomic variation and its impact on gene expression in Drosophila melanogaster. PLoS Genet 2012, 8:e1003055. 
10. Fan B, Onteru SK, Du ZQ, Garrick DJ, Stalder KJ, Rothschild MF: Genome-wide association study identifies loci for body composition and structural soundness traits in pigs. PLOS ONE 2011, 6:e14726.

11. Wang JY, Luo YR, Fu WX, Lu X, Zhou JP, Ding XD, Liu JF, Zhang Q: Genome-wide association studies for hematological traits in swine. Anim Genet 2013, 44:34-43.

12. Fu WX, Liu Y, Lu X, Niu XY, Ding XD, Liu JF, Zhang Q: A genome-wide association study identifies two novel promising candidate genes affecting Escherichia coli F4ab/F4ac susceptibility in swine. PLOS ONE 2012, 7:e32127.

13. Chen C, Yang B, Zeng Z, Yang H, Liu C, Ren J, Huang L: Genetic dissection of blood lipid traits by integrating genome-wide association study and gene expression profiling in a porcine model. BMC Genomics 2013, 14:848.

14. Yang B, Zhang W, Zhang Z, Fan Y, Xie X, Ai H, Ma J, Xiao S, Huang L, Ren J: Genome-wide association analyses for fatty acid composition in porcine muscle and abdominal fat tissues. PLOS ONE 2013, 8:e65554

15. Guo Y, Mao H, Ren J, Yan X, Duan Y, Yang G, Ren D, Zhang Z, Yang B, Ouyang J, Brenig B, Haley C, Huang L: A linkage map of the porcine genome from a large-scale White Duroc $x$ Erhualian resource population and evaluation of factors affecting recombination rates. Anim Genet 2009, 40:47-52.

16. Ma J, Yang J, Zhou L, Zhang Z, Ma H, Xie X, Zhang F, Xiong X, Cui L, Yang $H$, Liu X, Duan Y, Xhiao S, Ai H, Ren J, Huang L: Genome-wide association study of meat quality traits in a White DurocxErhualian F2 intercross and Chinese Sutai pigs. PLOS ONE 2013, 8:e64047.

17. Zhang Z, Hong Y, Gao J, Xiao S, Ma J, Zhang W, Ren J, Huang L: Genome-wide association study reveals constant and specific loci for hematological traits at three time stages in a White Duroc x Erhualian F2 resource population. PLOS ONE 2013, 8:e63665.

18. Purcell S, Neale B, Todd-Brown K, Thomas L, Ferreira MA, Bender D, Maller J, Sklar P, de Bakker PI, Daly MJ, Sham PC: PLINK: a tool set for wholegenome association and population-based linkage analyses. Am J Hum Genet 2007, 81:559-575.

19. R core team: R: A Language and Environment for Statistical Computing. Vienna, Austria: R foundation for statistical computing; 2013.

20. Aulchenko YS, Ripke S, Isaacs A, van Duijn CM: GenABEL: an R library for genome-wide association analysis. Bioinformatics 2007, 23:1294-1296.

21. Churchill GA, Doerge RW: Empirical threshold values for quantitative trait mapping. Genetics 1994, 138:963-971.

22. Storey JD: A direct approach to false discovery rates. $J R$ Stat Soc Series $B$ 2002, 64:479-498.

23. van Nas A, Guhathakurta D, Wang SS, Yehya N, Horvath S, Zhang B, Ingram-Drake L, Chaudhuri G, Schadt EE, Drake, Arnold AP, Lusis AJ: Elucidating the role of gonadal hormones in sexually dimorphic gene coexpression networks. Endocrinology 2009, 150:1235-1249.

24. Goodnight SH Jr, Harris WS, Connor WE, Illingworth DR: Polyunsaturated fatty acids, hyperlipidemia, and thrombosis. Arteriosclerosis 1982, 2:87-113.

25. Wray NR, Yang J, Hayes BJ, Price AL, Goddard ME, Visscher PM: Pitfalls of predicting complex traits from SNPs. Nat Rev Genet 2013, 14:507-515.

26. Zillikens MC, Yazdanpanah M, Pardo LM, Rivadeneira F, Aulchenko YS, Oostra BA, Uitterlinden AG, Pols HA, van Duijn CM: Sex-specific genetic effects influence variation in body composition. Diabetologia 2008, 51:2233-2241

27. Lindahl G, Lundstrom K, Tornberg E: Contribution of pigment content, myoglobin forms and internal reflectance to the colour of pork loin and ham from pure breed pigs. Meat Sci 2001, 59:141-151.

28. Te Pas MF, Keuning E, Hulsegge B, Hoving-Bolink AH, Evans G, Mulder HA: Longissimus muscle transcriptome profiles related to carcass and meat quality traits in fresh meat Pietrain carcasses. J Anim Sci 2010, 88:4044-4055

29. Taniguchi M, Hayashi T, Nii M, Yamaguchi T, Fujishima-Kanaya N, Awata T, Mikawa S: Overexpression of NUDT7, a candidate quantitative trait locus for pork color, downregulates heme biosynthesis in $L 6$ myoblasts. Meat Sci 2010, 86:728-732.

30. Barua S, Kuizon S, Chadman KK, Flory MJ, Brown WT, Junaid MA: Single-base resolution of mouse offspring brain methylome reveals epigenome modifications caused by gestational folic acid. Epigenetics Chromatin 2014, 7:3

31. Heid IM, Jackson AU, Randall JC, Winkler TW, Qi L, Steinthorsdottir V, Thorleifsson G, Zillikens MC, Speliotes EK, Magi R, Workalemahu T, White CC, Bouatia-Naji N, Harris TB, Berndt SI, Ingelsson E, Willer CJ, Weedon MN, Luan J,
Vedantam S, Esko T, Kilpeläinen TO, Kutalik Z, Li S, Monda KL, Dixon AL, Holmes CC, Kaplan LM, Liang L, Min JL: Meta-analysis identifies 13 new loci associated with waist-hip ratio and reveals sexual dimorphism in the genetic basis of fat distribution. Nat Genet 2010, 42:949-960.

32. McDaniell R, Lee BK, Song L, Liu Z, Boyle AP, Erdos MR, Scott LJ, Morken MA, Kucera KS, Battenhouse A, Keefe D, Collins FS, Willard HF, Lieb JD, Furey TS, Crawford GE, lyer VR, Birney E: Heritable individual-specific and allele-specific chromatin signatures in humans. Science 2010, 328:235-239.

doi:10.1186/s12711-014-0076-2

Cite this article as: Cui et al: Sexually dimorphic genetic architecture of complex traits in a large-scale $F_{2}$ cross in pigs. Genetics Selection Evolution 2014 46:76.

\section{Submit your next manuscript to BioMed Central and take full advantage of:}

- Convenient online submission

- Thorough peer review

- No space constraints or color figure charges

- Immediate publication on acceptance

- Inclusion in PubMed, CAS, Scopus and Google Scholar

- Research which is freely available for redistribution

Submit your manuscript at www.biomedcentral.com/submit
C) Biomed Central 